\title{
reCHERches
}

Culture et histoire dans l'espace roman

$25 \mid 2020$

Mentir au théâtre

\section{El Arroyo de los Ángeles}

\section{Miguel Palacios}

\section{(2) OpenEdition}

\section{Journals}

\section{Edición electrónica}

URL: https://journals.openedition.org/cher/412

DOI: 10.4000/cher.412

ISSN: 2803-5992

\section{Editor}

Presses universitaires de Strasbourg

\section{Edición impresa}

Fecha de publicación: 19 noviembre 2020

Paginación: 192-201

ISBN: 979-10-344-0071-3

ISSN: 1968-035X

\section{Referencia electrónica}

Miguel Palacios, «El Arroyo de los Ángeles», reCHERches [En línea], 25 | 2020, Publicado el 01 octubre 2021, consultado el 17 noviembre 2021. URL: http://journals.openedition.org/cher/412 ; DOI: https:// doi.org/10.4000/cher.412 


\section{El Arroyo de los Ángeles \\ Version espagnole}

CARMELa y Macu, en los sesenta y muchos las dos.

En la cocina de CARMela. Pequeña, limpia, coqueta; típica cocina de familia andaluza de clase media.

Carmela. - ¿Te lo puedes creer, Macu? El hombre me da dinero de menos y, cuando lo aviso, se enfada y me dice con toda su cara que no es problema suyo, que cuando me pagó estaba bien.

Macu. - ¿No te lo ha dado?

Carmela.- Sí, al final, sí; pero ha sido muy violento. Yo ya no estoy para estos trotes.

Macu. - El que se ha equivocado ha sido él.

Carmela.- Ya sé, pero la culpa también es mía. Mi niño me tiene dicho que cuente el dinero delante del cliente, pero es que me da apuro. Si él ya lo ha contado, pues ya está. Hay que confiar en la gente.

Macu.- Tú eres demasiado buena, Carmela. Si fuese la primera vez, pero el año pasado te ocurrió lo mismo.

Carmela. - ¡Dos veces! Esta es la tercera, y lo peor es que no hay vuelta atrás: al final, cobro lo que falta, pero al cliente lo pierdo. Se sienten..., yo qué sé, como si los estuviese engañando.

MACU. - ¡No!

Carmela.- Sí, hija, sí. ¿Tú te crees que es justo?

Macu. - ¡Por supuesto que no!

Carmela.- Pues así está la cosa. Yo ya no estoy para trabajar, si no fuese por el niño... Desde que se murió Mateo he seguido con el negocio para llevar la casa para delante, pero yo no sé de estas cosas. ¿Tú me ves buscando clientes? ¡Si me cuesta la misma vida gestionar los que tengo! Pero entre los que ha hundido la crisis, los que se han jubilado y los que me han dejado por cosas como esta... No puedo perder ni uno más.

Macu.- Carmela, y no has pensado..., ya sé que suena feo, pero lo mismo el hombre tenía razón. Quiero decir, igual lo había contado bien y el pico que te ha faltado se te perdió aquí.

CARmela.- ¿Aquí? ¿Aquí cómo va a ser? ¿Cómo va a perderse el dinero en la casa?

MACU.- No sé; ya, es raro, pero tu niño... 


\section{Rue du Ruisseau des Anges 1 Version française}

CARMela et MaCU, toutes deux la soixantaine passée.

Cuisine de Carmela, typique d'une famille andalouse de la classe moyenne, petite, propre et joliment décorée.

CARMela. - Tu peux le croire, Macu? L'homme me donne de l'argent en moins, quand je le lui fais remarquer, il se met en colère, et, culotté comme pas un, il me dit que ce n'est pas lui qui s'est trompé, qu'il m’a donné le compte juste.

MACU.- Il ne t'a pas remis ce qui manquait?

CARMEla.- Si, finalement, si, mais ça a été vraiment violent pour moi. Je ne m'y fais pas, je n'ai plus l'âge pour ça.

Macu.- C'est lui qui s'est trompé.

Carmela.- Je le sais, mais c'est aussi de ma faute. Mon gamin me le répète sans cesse, il faut que je compte l'argent devant le client, mais, j'ai des scrupules à le faire. S'il a déjà compté, eh bien c'est bon. Il faut faire confiance aux gens.

Macu. - Tu es trop bonne, Carmela. Si c'était la première fois mais, l'année dernière, ça t'est déjà arrivé.

Carmela.- Deux fois! Ça c'est la troisième, et le pire c'est qu'il n'y a pas de marche arrière possible: à la fin, j'encaisse bien ce qui manque, mais je perds le client. Ils ont l'impression..., je ne sais pas, comme si on les grugeait.

MACU.- Non!

CARMELA.- Si, ma fille, si. Tu crois ça juste?

MACU.- Bien sûr que non!

Carmela.- Eh bien, on en est là. Moi, je ne suis plus faite pour ça. S’il n'y avait pas mon gamin... Quand Mathieu est mort, j'ai poursuivi l'activité, il fallait bien s'en sortir, mais je n'y connais pas grand-chose. Tu me vois chercher des clients, alors que je galère avec ceux que j'ai déjà? Mais avec la crise qui en a ruiné plus d'un, ceux qui ont pris leur retraite et ceux qui ne viennent plus après des couacs comme celui-ci... Je ne peux pas me permettre d'en perdre un seul de plus.

MAcu.- Carmela, ça ne t'est pas passé par la tête... je sais que c'est une mauvaise pensée..., que l'homme avait raison. Je veux dire que, peut-être, il t'a bien donné le compte juste et c'est toi qui a perdu ce qui manquait, ici.

Carmela.- Ici? Ici, comment ça? Comment veux-tu que je perde de l'argent à la maison?

MACU. - Je ne sais pas, oui, c'est quand même bizarre, mais ton fils...

Traduction de Isabelle Reck.

1 Note de la traductrice: "El Arroyo de los Ángeles" est le nom d'une avenue et d'un quartier de la commune de Miraflores de los Ángeles, de la ville de Málaga. Nous avons fait le choix de le traduire pour en garder le sens métaphorique dans le contexte de la pièce. 
CARmela.- ¿Mi niño? ¿No estarás insinuando...? ¡Ay, Macu, no! Mi niño tiene muchos defectos, pero él no sería capaz... ¡A su madre, no! ¡Ni a nadie! Eso fue en la época mala, cuando no era él. Lleva limpio año y medio, si hasta tiene trabajo y todo, de conserje, en el Sagrado Corazón, tú lo sabes. Él no le haría eso a su madre. Estando bien, no.

MAcu.- ¿ No tienes una caja de seguridad o algo así?

Carmela.- Sí, ahí, en el salón, a la espalda del Cautivo ${ }^{1}$, pero es un lío.

Macu.- ¡Chiquilla, pero mételo ahí! Ahórrate penas.

Carmela.- Otra vez, ¡que no, que el hombre se equivocó al contarlo! Mi Joaquín es un buen niño.

Macu._- Lo que tú digas. ¡Venga!, vamos a tomarnos un cafelito; verás como te sientes mejor.

Carmela.- Sí, eso, un cafelito... Es que es un follón.

MACU. - ¿El qué?

CARMEla.- Guardar el dinero en la caja fuerte.

MACU._ ¡Ah!, sigues con eso.

CARMela.- Le tengo los números bloqueados, porque, como se cambien, luego, para volver a ponerlos, ni te imaginas el follón.

Macu.- Ya, bueno, da igual, no le des más vueltas. Toma, el café; con dos de estevia, como a ti te gusta.

CARMela.- ¡Uy! A mí como me gusta es con tres cucharaditas de azúcar, pero, hija, así de puñetera es la vida. ¡Qué le vamos a hacer! Tendremos que amoldarnos a lo que nos ha tocado.

Macu. - Ni que lo digas, comadre, ni que lo digas.

Dan un par de sorbos sin decir una palabra.

Macu.-Pero... No, que estaba pensando. Si le dejas los números bloqueados, ¿por qué es un follón? La abres, metes el dinero y vuelves a cerrarla, ¿no?

CARmela.- ¡Ay!, por la llave. La guardo detrás del mueble del salón. Tengo que mover el mueble, coger la llave, quitar el cuadro, abrir la caja...

MACU.- ¿Y tu niño lo sabe?

CARMEla. - ¿El qué?

MACU.- Que la llave está ahí.

CARMela.- ¡Claro que lo sabe!

1 Se trata de la estatua de "Cristo cautivo", tan popular y venerada en Málaga. Obra de 1938 del escultor José Gabriel Miguel Simón. 
Carmela.- Mon gamin? Tu n'insinuerais pas que...? Bon sang, Macu, non! Mon fils a beaucoup de défauts, mais il n'en serait pas capable... Voler sa propre mère! Non! Il ne volerait personne! Ça c'était avant, à l'époque où il n'était pas lui-même. Il n'a rien pris depuis un an et demi, et il a même trouvé un travail, comme concierge, au Sacré Cœur, ça tu le sais. Il ne ferait pas une telle chose. Pas maintenant qu'il a décroché, non.

MACU.- Tu n'as pas un coffre-fort ou quelque chose comme ça?

Carmela.- Oui, dans le salon, derrière le "Jésus-Christ garroté»", mais c'est compliqué.

MACU. - Eh bien alors, ma belle, utilise-le, et tu t'éviteras des problèmes.

Carmela.- Eh puis, non, je te le répète, non! L'homme s'est trompé dans ses comptes. Mon Joachim est un brave gamin.

Macu.- Comme tu veux. Allez, prenons un petit café, tu verras comme tu te sentiras mieux après.

Carmela.- Oui, un petit café... C’est que c'est compliqué!

MACU.- Quoi donc?

CARMEla.- Garder l'argent dans le coffre-fort.

MACU.- Ah, tu en es encore là.

Carmela. - J'ai bloqué les chiffres du code, car si jamais on les change, ensuite, pour les entrer à nouveau, je ne te raconte pas comme c'est compliqué.

Macu.- Bon, oui, ça ne fait rien, ne te prends pas plus la tête avec ça. Tiens, voici le café, avec deux sucrettes, comme tu l'aimes.

Carmela.- Tu sais, ma fille, c'est plutôt avec trois cuillères de sucre que je le préfère, moi, mais avec cette saleté de vie... Qu'est-ce qu'on peut y faire! Il faut bien qu'on se contente de ce qu'elle nous donne.

MACU._ À qui tu le dis, ma fille, à qui tu le dis.

Elles boivent quelques gorgées sans rien dire.

MACU.- Mais... voilà, je me disais, si tu bloques les numéros, pourquoi ce serait compliqué? Tu l'ouvres, tu y mets l'argent et tu le refermes, et voilà. Non?

CARMela.- Eh bien, à cause de la clé. Je la garde derrière le meuble du salon. Je dois déplacer le meuble, prendre la clé, décrocher le tableau, ouvrir le coffre-fort...

MACU. - Et ton gosse le sait?

CARMela.- Quoi?

MACU.- Que la clé est là.

CARmela.- Bien sûr qu'il le sait!

Macu.- Mais comment ça... ? Pourquoi? Après ce qu'il a fait la dernière fois, quand il a même vendu la télé!

Carmela.- Je te le répète, il est clean depuis un an et demi.

2 Il s'agit d'un tableau représentant "El Cautivo", statue du "Christ garroté" vénérée à Malaga, œuvre de 1938 de José Gabriel Miguel Simón. 
Macu.- Pero, ¿cómo...? ¿Por qué? Después de lo de la última vez, ¡que vendió hasta la tele!

CARMela.- - Te repito que lleva limpio un año y medio.

MAcU.- ¡Vale! ¡Vale! Tu Joaquín es un buen niño, ¡ya lo sé! ¡Vale! Da igual, si, de todas formas, el dinero no lo pones ahí.

Carmela.- El de los clientes no, prefiero dejarlo en el cajón del escritorio y de ahí al banco o se lo doy al repartidor para que se lo lleve directamente a fábrica. En la caja tengo los papeles del piso y algunos ahorrillos, por eso el niño tiene que saberlo. ¿Y si me ocurre algo? Los años no pasan en balde. Por lo menos, con ese dinero y el piso, estará cubierto.

Macu. - ¡De todas formas, Carmela! No te digo que lo dejes de lado, es tu hijo, yo lo entiendo. Lo que te digo es que te protejas; que confíes en alguien, en tu hermana o en cualquiera de tus primos...

CARMEla._-¡Mis primos! ¡De momento! ¡Con lo gamberros que son! Esos sí que son capaces de abrir la caja y fundirse los treinta mil euros en mujeres y bebida. ¡Que no veas tú cómo les gusta el alpiste!

MACU.— ¡Qué exagerada eres!

Carmela.- Tú no los conoces.

Macu.- Será eso, pero algo tienes que hacer. El niño es un buen chico, pero así no lo ayudas. Yo no te digo que lo acuses de nada, pero por lo menos protégete $y$, como tú dices, que disfrute ese dinero cuando faltes. Seguro que, para entonces, dentro de muchos años, Dios lo quiera, habrá echado cabeza.

CARMELA se queda un instante mirando por la ventana.

CARmela.- Bueno, lo que vamos a hacer, si a ti te parece bien, es guardar la llave de la caja fuerte en tu casa.

MACU.- No, Carmela, no. ¡Qué responsabilidad! ¿Y si alguien encuentra la forma de abrir la caja? No quiero ni pensarlo. Con guardarte la de la casa ya tengo bastante.

CARMEla.- ¡AAnda, no digas tonterías! ¿Cuántos años hace que vivimos puerta con puerta, cuarenta?

MACU._ ¡Y cincuenta!

CArmela.- Toda una vida. ¡Hemos pasado tanto! Hemos visto crecer a los niños, hemos enviudado, hemos superado lo de mi Joaquín, el divorcio de tu Sara, y cuando tu Jose estuvo dos años en el paro. Hemos sobrevivido a, ¿cuántas?, ¿cuatro crisis económicas?

MACU. - ¡Ay, si solo fuesen cuatro! ¿Cuándo hemos dejado nosotras de estar en crisis?

Carmela.- Y aquí seguimos. Por eso te digo, si no puedo fiarme de ti, ¿de quién me voy a fiar? Llevamos toda la vida bregando juntas.

MAcu. - Es verdad, hija, a las duras y a las maduras. Mira, ¡vale!, yo te la guardo. ¡Eso!, la pongo con la de la casa y cuando te haga falta, me la pides. 
Macu.- D'accord, d'accord! Ton Joachim est un brave garçon. Oui, je le sais! D’accord! Ça ne fait rien, puisque de toutes façons tu ne gardes pas là ton argent.

CARmela.- - Non, pas l'argent des clients, je préfère le garder dans le tiroir du secrétaire, et ensuite, direct à la banque, ou alors je le remets au coursier pour qu'il l'amène à l'usine. Dans le coffre-fort, je range les papiers de l'appartement et quelques petites économies, c'est pour ça qu'il faut que le gamin soit au courant. Car s'il m'arrive quelque chose? Je me fais vieille. L'appartement et cet argent le mettront à l'abri du besoin.

Macu.- De toute façon, Carmela! Je ne te dis pas de l'écarter, c'est ton fils, je le comprends. Ce que je dis c'est que tu dois te protéger, t'appuyer sur quelqu'un, sur ta sœur ou l'un de tes cousins, n'importe lequel...

CARmela.- Mes cousins! Alors là, de vrais voyous! Ils sont capables d'ouvrir le coffre-fort et de dilapider les trente mille euros en femmes et boisson. Si tu savais à quel point ces oiseaux raffolent de la gnôle.

MACU. - Tu exagères!

CARMela.- Tu ne les connais pas.

Macu.- Probablement, mais il faut bien que tu fasses quelque chose. Le gosse est un bon gamin, mais c'est pas comme ça que tu l'aides. Je ne dis pas de l'accuser de quoi que ce soit, mais au moins protège-toi, et, comme tu le dis toi-même, qu'il puisse profiter de cet argent quand tu ne seras plus là. Et je suis sûre qu'alors, dans très longtemps si Dieu le veut, il se sera assagi.

CARMELA regarde un moment par la fenêtre.

CARMELA.- Bon, ce qu'on va faire, si ça te semble bien, c'est que tu gardes chez toi la clé du coffre-fort.

Macu.- Non, Carmela, non. Quelle responsabilité! Et si jamais quelqu'un trouve le moyen d'ouvrir le coffre-fort? Je n'ose même pas y penser! J'ai la clé de ta maison, et c'est déjà une sacrée responsabilité.

CARmela.- Allons, ne dis pas de bêtises! Ça fait combien d'années que nous vivons tout près, l'une de l'autre, nos deux portes côte à côte, quarante?

MACU.- Même cinquante!

Carmela.- Toute une vie! On a vécu tant de choses! On a vu grandir nos enfants, on est devenues veuves en même temps, on a surmonté le problème de mon Joachim, le divorce de ta Sara et les deux ans de chômage de ton Joseph. On a survécu à combien? Quatre crises économiques?

Macu.-Ah, si c'était seulement quatre! On n'a pas connu que ça, des crises?

CARMEla.- Oui, et on est toujours là. C'est pourquoi, je te le dis, si je ne peux pas te faire confiance à toi, à qui le pourrais-je? On s'est battues ensemble toute notre vie.

Macu.- C'est vrai, ma fille, contre vents et marées. Bon, d'accord, je la garde ta clé. Je la mets avec la clé de chez toi et quand tu en auras besoin, tu me la demandes. 
CARMela.- Espera, que voy a por ella.

Va a salir, pero a mitad de camino da marcha atrás.

CARMela.- ¡Seré tonta, Dios mío!

MACU. - ¿Qué pasa?

CARmela.- Que me acabo de acordar que le tengo que pagar mañana al informático.

MACU.— ¿Al...? ¿Qué? ¿Y qué negocio tienes tú con un informático? Si no tienes ni ordenador.

CARMEla.- - Verás, no sé si es informático, informático. El de la camarita de seguridad... Si te lo dije.

MACU.— ¿Qué...? De eso no me has dicho nada tú.

Carmela.- ¡Claro que sí!, el año pasado, cuando me faltó dinero la segunda vez.

Macu.- ¿Ah, sí?... ¡Sí, es verdad! Ahora me acuerdo, pero dijiste que ibas a buscar a alguien que te pusiese una cámara, no que la hubieses puesto ya.

Carmela.- No, si no la puse.

MACU. - ¿Y entonces?

CARMEla.- - La he puesto este año, ahí, en el salón.

MACU.- Frente al Cautivo.

CARmela.- ¡Qué va! Ya te lo he dicho, de la caja de seguridad no ha faltado nunca nada. La he puesto justito frente al cajón del escritorio.

MACU. - Ya, donde guardas...

Carmela. - ... el dinero de los clientes. ¡Eso es!

MACU. - ¡Ah!

CARmela.- Sí, es curiosísimo. La cámara graba día y noche y lo envía todo a Madrid, a un ordenador al que está conectada; no me preguntes cómo, que no lo sé... Allí se queda quince días y, si pasa algo, pues se miran las grabaciones, y si no, se borran y ya está.

MACU.- ¡Ah!

Carmela.- Sí, está bien, ¿verdad? ¡Que tengamos que llegar a esto! Por eso te digo, yo sé que mi niño no ha cogido lo que falta.

MACU. - No.

Un instante de silencio en el que MACU mira al suelo y CARMELA la mira a ella.

CARMEla.- Bueno, pues mañana te doy la llave. Mira, y así aprovecho también para darte la nueva de la casa, que, con lo que ha pasado, he aprovechado para cambiar la cerradura, que nunca se sabe...; pero, ¡qué estoy diciendo! ¡Qué tontería! Fíjate, ya estoy creyéndome lo que no es. ¡Óyeme, Macu! En esta casa no hay ningún ladrón. El hombre se equivocó al contar el dinero. MACU.- Sí, se equivocó, o..., ¿quién sabe?, la cosa está tan mala.

Carmela.- Sí, hija, está mala para todas. ¡Qué se le va a hacer! Pero, ¡verás!, que con ese dinero tampoco sale nadie de pobre.

MACU.- Ya. 
CARmela.- Attends, je vais la chercher.

Elle est sur le point de sortir, mais se ravise et revient sur ses pas.

Carmela.- Mais, suis-je bête, mon Dieu!

Macu.- Qu'est-ce qu'il y a?

CARMEla.- Je viens de me souvenir que, demain, je dois payer l'informaticien. Macu.- Qui ça? Qui ? Qu'est-ce que tu as à voir avec un informaticien? Tu n'as même pas un ordinateur.

Carmela.- Pour tout te dire, je ne sais pas si c'est vraiment un informaticien. C'est le gars de la petite caméra de surveillance... mais je t'en ai déjà parlé. MACU.- Quoi...? Ça, non, tu ne m'en as jamais parlé.

CARMela.- Bien sûr que si! L'année dernière, lorsque de l'argent avait encore disparu, pour la deuxième fois.

MAcu._- Ah, oui! Oui, c'est vrai! Je me souviens maintenant, mais tu avais dit que tu allais chercher quelqu'un pour t'installer la caméra, pas que tu l'avais déjà fait.

CARmela.- Non, je ne l'avais pas fait installer.

MACU.- Et alors?

CARMela.- Je l'ai installée cette année, là, dans le salon.

MACU.- Face à «Jésus-Christ garroté»?

Carmela.- Non, pas du tout. Je te l'ai déjà dit, rien n'a jamais disparu du coffre-fort. Je l'ai orientée juste pile vers le tiroir du secrétaire.

MACU.- Oui, là où tu gardes...

Carmela. - ...l'argent des clients. C'est bien ça!

MACU.-Ah!

CARmela.- Oui, c'est très marrant! La caméra enregistre nuit et jour et envoie le tout à Madrid, à l'ordinateur auquel elle est connectée. Ne me demande pas comment ça marche, je n'en sais rien... Là-bas, ils gardent les enregistrements quinze jours et, s'il y a quelque chose, on les regarde, sinon, on les efface.

MACU.-Ah!

Carmela.- Oui, c'est bien, pas vrai? Mais, tout de même, en devoir arriver là ! C'est pour ça que je te dis que je sais que mon gamin n'a pas pris ce qui manque.

MACU. - D'accord.

Un instant de silence. MACU regarde le sol et CARMELA la regarde, elle.

Carmela.- Bon, alors, demain je te donne la clé. Et, comme ça, j’en profite pour te donner aussi la nouvelle clé de chez moi, car après toute cette histoire, j'ai fait remplacer la serrure, on ne sait jamais... mais, qu'est-ce ce que je dis! Quelle sotte je fais! Tu te rends compte, je commence à croire ce qui n'est pas. Écoute, Macu, j'en suis sûre et certaine, dans cette maison, il n'y a aucun voleur. C'est l'homme qui s'est trompé en comptant l'argent. Macu.- Oui, il s'est trompé, ou, qui sait, les temps sont si durs. 
CARmela.- ¡Venga, anímate! Coge el abrigo y nos vamos al Cayetano a merendar.

MACU.- Yo...

CARmela.- No me digas que no te apetecen unos churros.

MACU. - ¿Con chocolate?

Carmela.- Con chocolate.

MaCU.- Es que...

CARMela. - ¿Sí?

MACU. - ... este mes ando algo corta.

Carmela. - No pasa nada, invito yo. A las duras y a las maduras, Macu; a las duras y a las maduras.

Oscuro.

Sevilla, abril 2020 
CARmela.- Oui, ma fille, les temps sont durs pour nous toutes. Qu'est-ce qu'on y peut! Mais, bon, les petits ruisseaux ne font pas toujours les grandes rivières.

MACU.- C'est bien vrai.

Carmela.- Allez, courage! Prends ton manteau et allons goûter au Cayetano.

MACU.- Moi, je.

Carmela. - Ne me dis pas que tu n'as pas envie de beignets.

MACU.- Avec du chocolat chaud?

Carmela.- Avec du chocolat chaud.

MaCU.- C'est que...

CARMEla.- Oui?

MACU. - ... ce mois-ci, je suis un peu juste.

Carmela.- Ne t'en fais pas, je t'invite. Contre vents et marées, Macu, contre vents et marées.

Obscurité.

Séville, avril 2020 